\title{
Bandas amnióticas constrictivas y derivación oportuna. A propósito de 2 casos clínicos
}

\author{
CARMEN GLORIA MOROVIC I. ${ }^{1}$, SUSANA SEARLE F. ${ }^{2}$, CLAUDIA VIDAL T. ${ }^{3}$ \\ 1. Cirujano Plástico, Facultad de Medicina, Universidad de Chile. Departamento de Unidad Plástica y Reconstructiva, \\ Hospital Luis Calvo Mackenna, Cirugía Plástica Infantil Clínica Las Condes. \\ 2 Residente de Cirugía Plástica y Reconstructiva, Facultad de Medicina, Pontificia Universidad Católica de Chile. \\ 3. Cirujano Infantil, Departamento de Cirugía Plástica y Reconstructiva, Hospital Luis Calvo Mackenna, Clínica Santa María.
}

\begin{abstract}
Constriction band syndrome and the right time for intervention. Two clinical cases
\end{abstract}

Introduction: The amniotic band syndrome is an infrequent affection, of controversial aetiology, with extreme heterogeneous manifestations. Extremities are most frequently compromised and may be associated to other malformations. These newborns rarely need immediate surgery nevertheless the surgical indication must be timely and not postponed, which could mean loss of the extremity. Objective: To report 2 patients with the amniotic band syndrome, in whom casual early interventions were associated to a favorable prognosis. Case reports: We present two patients with an important delay in the referral to the specialist because of the preconceived myths that either full growth was necessary for definitive surgery (case 2) or that no possible treatment could be offered due to the severity of the diagnosis (case 1). All constricting bands were successfully and effectively released with multiple $\mathrm{Z}$ plasties with uneventful post operative evolution. Conclusion: We discuss and emphasize the importance of early diagnosis and intervention.

(Key words: Amniotic band syndrome, constrictive band syndrome, congenital malformations).

Rev Chil Pediatr 2013; 84 (3): 318-322

\section{RESUMEN}

Introducción: El síndrome de bridas amnióticas (SBA) es una afección infrecuente de etiología controversial con manifestaciones muy heterogéneas. Las extremidades son las más frecuentemente afectadas, y puede asociarse a otras malformaciones. El recién nacido con SBA rara vez va a requerir de cirugía inmediata, sin embargo, la evaluación por el especialista y la indicación quirúrgica debe ser oportuna y no postergada innecesariamente, lo cual podría significar la pérdida de la extremidad. Objetivo: Comunicar 2 casos de SBA que afectan la extremidad superior, en los cuales la intervención oportuna providencial resultó en un favorable

Recibido el 15 de abril de 2012, devuelto para corregir el 30 de julio de 2012, segunda versión 14 de febrero de 2013, aceptado para publicación el 02 de abril de 2013.

Este trabajo cumple con los requisitos sobre consentimiento /asentimiento informado, comité de ética, financiamiento, estudios animales y sobre la ausencia de conflictos de intereses según corresponda.

Correspondencia a:

Dra. Carmen Gloria Morovic J.

E-mail:cmorovic@gmail.com 
pronóstico. Caso clínico: Reportamos dos casos, donde hubo una derivación tardía de los pacientes por mitos preconcebidos tales como "que era necesario completar el crecimiento para realizar un tratamiento definitivo" (caso 2) o "que no había ningún tratamiento que ofrecerle frente a la severidad de la malformación del recién nacido" (caso 1). En ambos pacientes las bridas constrictivas fueron liberadas en forma exitosa con el uso de múltiples zetoplastias, sin complicaciones en la evolución postoperatoria. Conclusión: Se discute y enfatiza la importancia del diagnóstico, derivación y tratamiento precoz de ésta patología.

(Palabras clave: Síndrome de bandas amnióticas, síndrome de bandas constrictivas, malformación congénita). Rev Chil Pediatr 2013; 84 (3): 318-322

\section{Introducción}

El Síndrome de Bandas Amnióticas (SBA) se caracteriza por disrupciones (bandas constrictivas, amputaciones y acrosindactilia), deformidades (fuerzas anormales que altera el desarrollo fetal normal), secundarias a oligohidroamnios por compresión del cuerpo fetal por las paredes uterinas y reducción de los movimientos fetales, y malformaciones. Teniendo en cuenta la asociación de éstas tres alteraciones y por tanto la heterogenicidad en la presentación de éste síndrome, se entiende la dificultad de consenso en cuanto a su etiología.

Han sido propuestas variadas teorías para explicar la fisiopatología del SBA, sin embargo ninguna por sí sola es capaz de explicar la totalidad de las tres asociaciones: Torpin ${ }^{1}$ (1965), describió la teoría exógena o extrínseca, donde se supone trauma y rotura del saco amniótico que conlleva la formación de bandas fibróticas amniocoriónicas mesodérmicas. Estas bandas fibróticas "flotarían" libremente en el líquido amniótico enredándose en los segmentos más prominentes como son las extremidades del feto. Esta banda constrictiva reduciría la circulación sanguínea hacia distal con la consecuente alteración congénita del feto. Esta teoría nos da una respuesta a la asimetría y la gran variedad de los hallazgos y nivel de amputación de las extremidades. La ruptura del saco amniótico conlleva una reducción del líquido amniótico con la disminución de los movimientos fetales y el aumento de la presión de las paredes uterinas sobre éste, lo que provocaría las deformidades.

Sin embargo, la alta incidencia de asociación a otras malformaciones y compromiso visceral en estos recién nacidos sugiere la pre- sencia de un factor "intrínseco", teoría preconizada por Streeter ${ }^{2}$ (1930), quién postula un desarrollo anormal en la línea germinal responsable del desarrollo de las bandas constrictivas. Esta teoría es adecuada en la explicación de las malformaciones craneofaciales mayores, viscerales, pero no las alteraciones a nivel de las extremidades.

Existe una tercera teoría, vascular, de Van Allen $^{3}$ (1981), capaz de explicar las anormalidades fetales tanto externas como internas. $\mathrm{Su}$ teoría describe la presencia de un evento traumático (amniocentesis, rotura amniótica) o teratógeno que interrumpe el aporte vascular fetal y conlleva necrosis y hemorragia. El momento de la injuria va determinar finalmente la manifestación clínica.

$\mathrm{Al}$ examen físico es característico el anillo constrictor circunferencial en extremidades que puede comprometer sólo el tejido blando o bien llegar hasta el plano óseo y asociarse a linfedema y/o compromiso vascular secundario. Estas características permiten clasificar la(s) lesion(es) en cuatro grupos según la Clasificación de Patterson ${ }^{4}$, considerando la severidad y el compromiso de la extremidad:1) anillo constrictivo simple, con una extremidad normal hacia distal; 2) anillo constrictivo con deformidad hacia distal de la extremidad, atrofia y linfedema; 3 ) brida constrictiva asociada a sindactilia (sindactilia fenestrada) y 4) amputación intra uterina. Esta clasificación tiene relevancia en el sentido que define conductas. Es así como los grados 2 y 3 deben ser derivados precozmente al cirujano para permitir un tratamiento quirúrgico oportuno, que en ocasiones puede significar la recuperación de la extremidad comprometida, incluso en casos que lo ameritan puede ser necesario su resolución en 


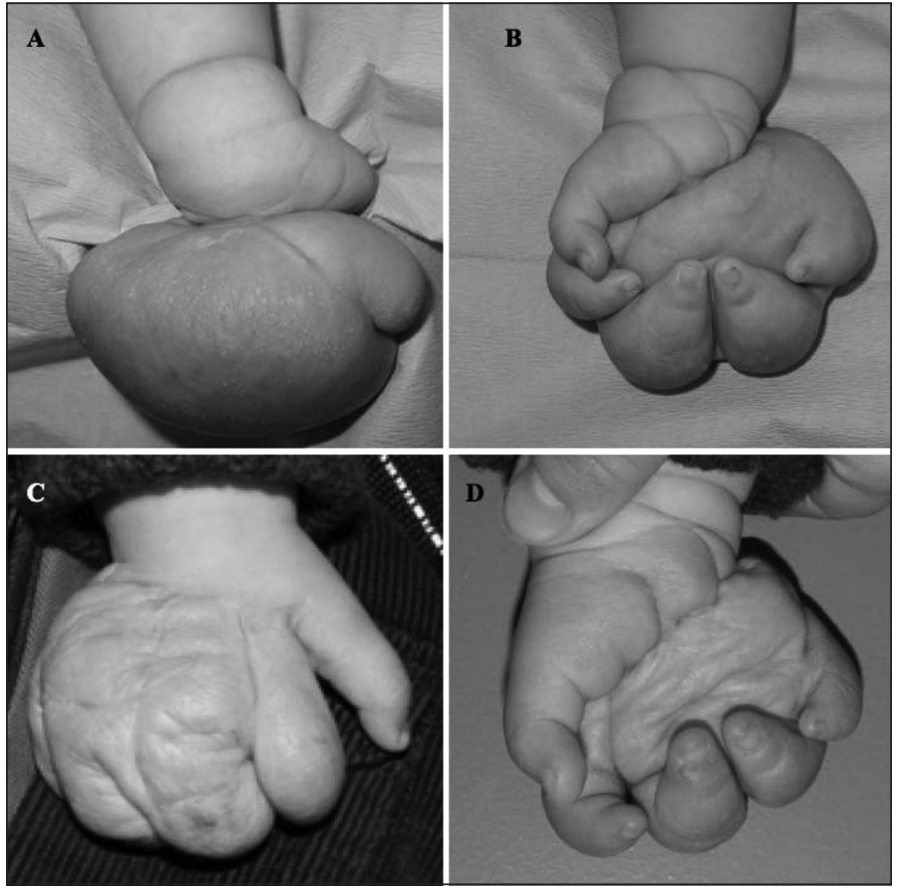

Figura 1. Vista preoperatoria dorsal (A) y palmar (B) de la banda constrictiva circunferencial media palmar C) y D) resultado post operatorio tardío con pronóstico estético-funcional adecuado a largo plazo. el período neonatal. En el caso de diagnóstico intrauterino y si las condiciones lo permiten, se puede considerar la cirugía fetal con el objeto de salvar la extremidad con riesgo de amputación o deformidad.

El objetivo de este reporte es presentar dos casos de SBA que comprometen la extremidad superior, con amenaza de pérdida del miembro por derivación tardía. Se discute consideraciones acerca del momento de la derivación y cirugía correctora.

\section{Caso clínico}

\section{Caso 1}

Niño de término, nacido en un hospital de provincia, derivado a la Unidad de Cirugía Plástica del Hospital Luis Calvo Mackenna a los cuatro meses de edad para manejo de su fisura labio-palatina. En su evaluación inicial en nuestra Unidad, impresionó la presencia de banda constrictiva a nivel medio palmar transversal en su mano derecha, con gran linfedema secundario y riesgo de amputación de la parte distal de su mano (figura 1 A y B). En su hospital de origen se había descartado cualquier alternativa de tratamiento, por considerarla no viable y estaba programada para amputación posterior. El paciente no presentaba otras alteraciones asociada a su brida amniótica y su fisura labio palatina bilateral. No tuvo diagnóstico prenatal ni antecedentes familiares de malformaciones o eventos adversos durante el embarazo. La radiografía simple de mano reveló conservación de las estructuras óseas pero con gran separación de los huesos de la mano entre sí. Dado el diagnóstico del paciente (brida amniótica grado 2 de Patterson), y su edad se consideró necesario realizar la liberación de las bridas en forma inmediata para intentar rescatar y salvar la extremidad. La cirugía consistió en resección circunferencial de la brida (por palmar y dorsal) tallándose múltiples zetoplastías, con lo que se logro disminución significativa del linfedema en el intraoperatorio (figura 1C). No hubo complicaciones relacionados con la vitalidad de colgajos ni lesiones neuro-vasculares. Dos meses después de la primera cirugía, en el momento del cierre del labio se realizó una reducción del tejido redundante derivado de la remisión del linfedema (figura 1D). 

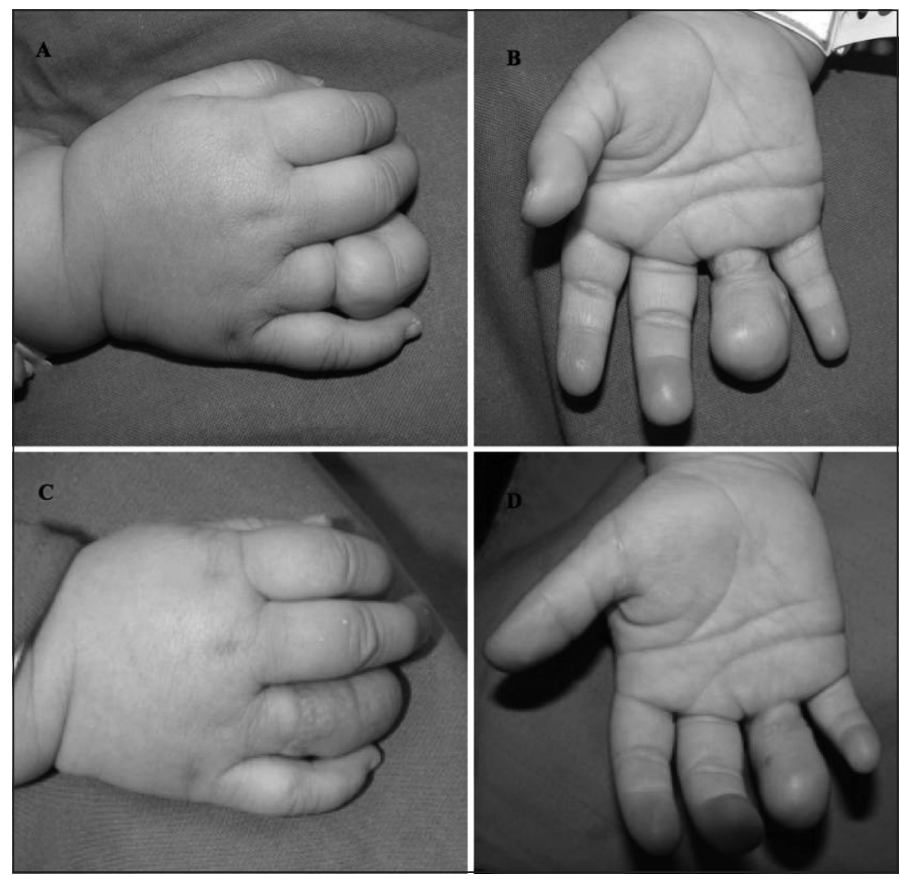

Figura 2. Vista preoperatoria palmar (A) y dorsal (B) de la banda amniótica circunferencial del dedo anular en reloj de arena, con linfedema importante; C) y D) vista a los tres meses post operatorio, con resolución del linfedema.

A dos años de seguimiento, ambas manos están prácticamente simétricas, con una ganancia progresiva del rango de movimiento y funcionalidad de la mano. A nivel digital existe cierta limitación a la extensión, secundaria a retracción de sus tendones, por lo que está en tratamiento kinésico activo y férula nocturna.

\section{Caso 2}

Paciente de sexo femenino, con brida amniótica circunferencial en la base de ambos dedos anulares, más severo a derecha, correspondiente al grado 2 de Patterson. En este dígito se observaba un anillo constrictivo en la base, con deformidad en reloj de arena por linfedema distal y amputación de la tercera falange (figura 2 A y B). La Rx simple de manos mostraba indemnidad de la primera y segunda falange, con ausencia de la tercera, además de engrosamiento importante de los tejidos blandos. La paciente no presentaba otras malformaciones asociadas. A pesar que en este caso el diagnóstico de SBA se hizo al nacimiento por su pediatra, este recomendó esperar hasta cumplir el año de edad para lograr mayor desarrollo antes de su corrección quirúrgica. Circunstancialmente el paciente consultó al cirujano por otro diagnóstico a los tres meses de edad, donde se indicó cirugía inmediata con técnica estándar (resección de la brida constrictiva y zetoplastias de descarga en un tiempo quirúrgico). Evolucionó sin complicaciones, pero con un dígito de mayor tamaño dada la redundancia de tejidos secundario al linfedema (figura $2 \mathrm{C}$ y D).

\section{Discusión}

En el año 1946 Stevenson describió el concepto de la liberación del anillo constrictivo, para mejorar la irrigación del segmento hacia distal, sin embargo, estudios posteriores acerca de la irrigación de la piel, demostraron que ésta dependía principalmente de perforantes músculo cutánea directas. Basado en esta premisa, no habría ningún riesgo en la liberación de la brida en un sólo tiempo quirúrgico ${ }^{5,6}$. Es más, la liberación de la brida constrictiva en sí facilita el retorno venoso y linfático disminuyendo el edema, lo que mejora la irrigación del tejido distal a la constricción así como a la piel subyacente.

Por lo cual el momento de la cirugía debe 
ser evaluado caso a caso por el cirujano plástico pediátrico. Si bien no son frecuentes los casos en que se requerirá de una cirugía en el período neonatal para salvataige de una extremidad en riesgo, es necesario tener en consideración el concepto que mientras más precoz la cirugía mejor será el resultado funcional (menor edema por estasia venosa y linfática), lo que facilita la movilidad e incorporación de la extremidad a las funciones habituales así como menor redundancia de tejidos blandos y compromiso tendinoso.

Por largo tiempo no se consideraba la resolución precoz por el mito de riesgo de lesión neuro-vascular en los recién nacidos y/o lactantes menores. Sin embargo, debemos pensar que el riesgo de pérdida o lesión de la extremidad es mucho mayor al no hacer nada. Actualmente, el mayor desarrollo tecnológico nos proporciona excelente apoyo de imágenes tales como el ecografía doppler, scanner con reconstrucción tridimensional, resonancia nuclear magnética, entre otras, que nos dará vasta información sobre las condiciones anatómicas de la extremidad. Por otra parte, el uso de magnificación y el manejo por un cirujano plástico pediátrico con expertise, nos permiten realizar estas cirugías con mínimo riesgo neuro-vascular y mejor pronóstico funcional.

En los casos presentados no hubo complicaciones derivados de la cirugía (alteraciones neuro-vasculares y/o retardo en la cicatrización) a corto ni largo plazo. Posiblemente ambos pacientes se habrían beneficiado con la liberación temprana de las bridas constrictivas ya que se habría evitado el gran linfedema que presentaba al momento del diagnóstico (sobretodo el caso 1), la redundancia de tejidos, los problemas de retracción de sus tendones flexores y la consecuente limitación funcional secundaria.

En nuestro medio hay dificultad con la derivación oportuna a los centros especializados posiblemente por falta de conocimiento del momento apropiado para su derivación o por considerar la lesión fuera del alcance quirúrgico. En este sentido sugerimos derivar al pa- ciente lo antes posible y que sean los centros con experiencia los que recomienden el momento quirúrgico oportuno.

\section{Conclusiones}

Con la aplicación de la clasificación de Patterson, ya nos orienta hacia una conducta. Los grados extremos, 1 y 4, no tienen indicación de cirugía; mientras que los pacientes en grados 2 y 3 deben ser derivados a la brevedad ya que podrían beneficiarse con una intervención precoz. El momento exacto de la cirugía es crítico en estos pacientes, ya que determinará la posibilidad de salvar la extremidad y su futuro funcional.

La gran diversidad de asociaciones en estos defectos van a requerir de evaluación y manejo por un equipo multidisciplinario, (cirujano plástico pediátrico, genetista, traumatólogo y un equipo de rehabilitación), siendo esta evaluación inicial la que coordinara y determinara los tiempos en el tratamiento, que será fundamental en los resultados estético-funcionales que se logren a largo plazo.

\section{Referencias}

1.- Torpin L: Amniochorionic mesoblastic fibrous string and amniotic bands: associated constricting fetal malformations or fetal death. Am J Obstet Gynecol 1965; 91: 65-75.

2.- Streeter GL: Focal deficiencies in fetal tissues and their relation to intrauterine amputations. Contrib embryol Carnegie Inst 1930; 22: 1-44.

3.- Van Allen MI: Fetal vascular disruptions: mechanisms and some resulting birth defects. Paediatrics Ann 1981; 10: 219-33.

4.- Patterson TJS: Congenital ring constrictions. Br J Plast Surg 1961; 14: 1- 11.

5.- Choulakian MY, Williams HB: Surgical correction of congenital constriction band syndrome in children: Replacing z-plasty with direct closure. The Canadian Journal of Plastic Surgery 2008; 16 (4): 221-3.

6.- Kawamura K, Chung KC: Constriction band syndrome. Hand Clin 2009; 25 (2): 257-64. 